\title{
Facial myokymia: a clue to the diagnosis of multiple sclerosis
}

\author{
K. L. DE Silva \\ M.B.,B.S., M.R.C.P.
}

\author{
John Pearce \\ M.D., M.R.C.P.
}

\author{
Combined Neurological Service, \\ Hull Royal Infirmary, Hull
}

\begin{abstract}
Summary
Three patients with multiple sclerosis in whom facial myokymia occurred are described. The clinical and electromyographic features of this phenomenon are reviewed. The phenomenon is most frequently associated with multiple sclerosis, and when it is transient and unassociated with progressive facial paresis and other signs of a pontine tumour, is a strong indication of this condition.
\end{abstract}

\section{Introduction}

Facial myokymia is an unusual form of involuntary movement of the facial musculature which is characterized by spontaneous undulating waves of contracting muscle fibres spreading across the face.

In a recent review, Gutman, Thompson \& Martin (1969) were able to find forty cases reported in the world literature and added two of their own. Of these cases, in twenty-three the diagnosis of multiple sclerosis seemed 'well established, or at least quite likely' and at least ten had intrinsic pontine tumours. Thus, over half the reported cases have been associated with multiple sclerosis.

The purpose of this paper is to (1) assess the incidence of facial myokymia in multiple sclerosis, (2) report three further patients with this rare syndrome, and (3) record the results of electrophysiological investigations.

\section{Observations}

The approximate incidence of this phenomenon in multiple sclerosis can be inferred from the data in Table 1.

This shows the number of new cases of multiple sclerosis diagnosed in the Combined Neurological

TABLE 1

\begin{tabular}{lr}
\hline Total new cases & 103 \\
New cases per year & 26 \\
Cases with facial myokymia & 3 \\
Approximate frequency of facial & \\
$\quad$ myokymia in the cases of multiple & 1 in 34 \\
sclerosis & 1 in \\
\hline
\end{tabular}

Requests for reprints: Dr K.L. de Silva, Combined Neurological Service, Hull, Royal Infirmary, Hull, U.K.
Service, Hull Royal Infirmary, serving a population of approximately 500,000 during the period July 1967 to July 1971.

From these figures it is evident that facial myokymia does not seem to be as rare a phenomenon in multiple sclerosis as appears from the literature.

\section{Case reports}

Case 1 (H.R.I., 10100)

This 36-year-old sailor was admitted to the Combined Neurological Service, Hull Royal Infirmary, on 10 August 1967 with a history of episodes of sudden unsteadiness of gait and weakness of the legs lasting 1-3 months on four separate occasions at the ages of 16, 24, 31 and 34 years respectively. He had recovered fairly well from these episodes, but increasing weakness in both legs, clumsiness of the right arm and urgency incontinence had developed in the preceding 6 weeks and necessitated hospital admission. In the past he had had pulmonary tuberculosis. There was no family history of neurological illness.

On examination he was euphoric. There was jerky nystagmus on lateral gaze in either direction, pallor of the optic discs (acuity J8 in both eyes) but no other abnormality of the cranial nerves. There was considerable weakness in the right arm and marked ataxia in both arms, the right more than the left. Deep reflexes were exaggerated in both arms and legs. Abdominal reflexes were absent. He had a severe spastic paraparesis with exaggerated deep reflexes and bilateral extensor plantar responses. Vibration and position sense were absent in the right arm and both legs.

Routine laboratory tests including blood count and ESR, chest, cervical spine and skull X-rays, and EEGs showed no significant abnormality. His CSF manometrics were normal, protein $43 \mathrm{mg} / 100 \mathrm{ml}$, Lange 5433210000, WR negative. No treponemal antibodies were detected in either the blood or the CSF.

On the second day of admission he developed rhythmic, undulating involuntary movements, typical of facial myokymia, on the whole of the right side of his face, involving all the muscles on that side 
innervated by the facial nerve, from frontalis to platysma. This persisted continuously for 25 days, being present even during sleep, and then disappeared. The patient was unaware of its presence. During this time the right angle of the mouth was drawn up and there was narrowing of the right palpebral fissure. There was no evidence of any facial paresis.

Electromyography (EMG) using concentric needle electrodes showed continuous, rapidly recurring, spontaneous action potentials, amplitude $70-100 \mu \mathrm{V}$, firing at 75 bursts/sec, in the right orbicularis oris muscle with a tendency towards rhythmicity. For purposes of illustration, this was recorded using the electroencephalogram (EEG) and placing superficial electrodes over the facial muscles and recording between corresponding points on either side (Fig. 1). This recording and the EMG studies using concentric needle electrodes, showed a marked contrast between the two sides, with the continuous, rapidly recurring action potentials on the right side.

After a course of corticotrophin injections there was slight but definite improvement in the patient's condition. He later developed right-sided Jacksonian epilepsy 30 days after admission, followed by status epilepticus. This was controlled by intravenous anti-convulsants. Conventional EEG record- ings carried out after the onset of the epilepsy showed no spike or sharp wave activity but showed a low amplitude $50 \mu \mathrm{V}, 2 / \mathrm{sec}$, delta activity over the left fronto-parietal region. After recovery from this, he was able to recall occasional major epileptic attacks, abour four in all, which had occurred in the last 4 or 5 years. It seemed likely that his epilepsy was symptomatic of his multiple sclerosis (Matthews, 1962).

His condition gradually deteriorated at subsequent out-patient follow up. In April 1968 he was readmitted with focal motor epilepsy of the rightsided limbs which had subsequently become generalized. He had a post-epileptic right hemiplegia which resolved in $48 \mathrm{hr}$. A left carotid arteriogram was performed to exclude any other possible lesion, and was normal. He subsequently died of intercurrent infection in March 1969 in a geriatric ward, and an autopsy was not obtained. During the whole period of follow up there was no evidence of recurrence of the facial myokymia.

\section{Case 2 (H.R.I., 82315)}

This 47-year-old labourer was first seen as an outpatient at the Combined Neurological Service, Hull Royal Infirmary, on 6 January 1969, when he gave a

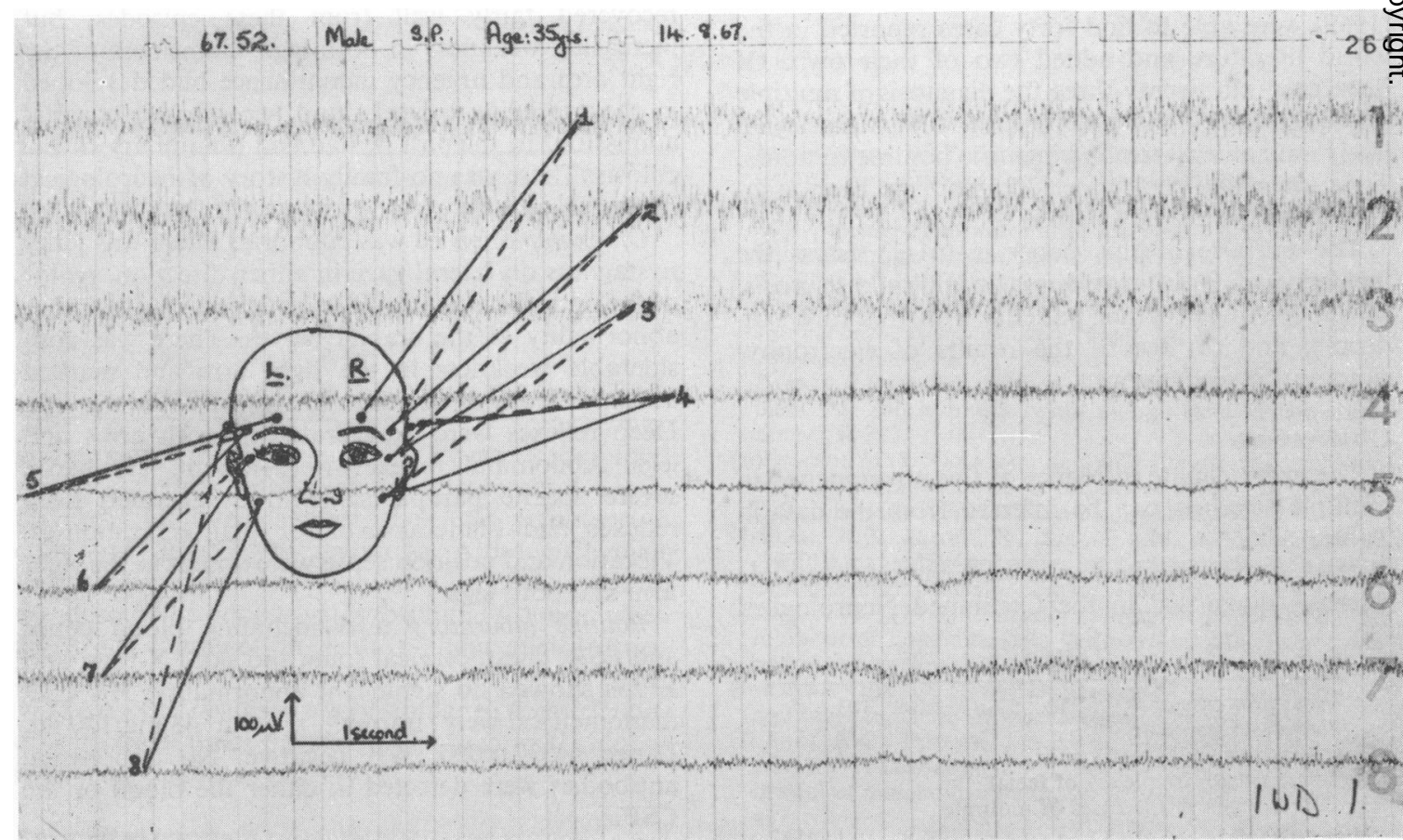

FIG. 1. Case 1. EEG record using surface electrodes over the facial muscles at corresponding points on either side, showing the contrast between the two sides. On the affected right side there are seen continuous, rapidly recurring spontaneous action potentials. 
history of intermittent attacks of numbness and tingling of the right foot and both arms and of clumsiness, especially on exertion of the left leg and both upper limbs, of 1 year's duration. Each attack lasted 2-3 weeks. During exertion, he also had noticed transient blurring of vision in the left eye. There was no history of recent bladder disturbance but he had had occasional faecal incontinence. He had had a detached retina in the right eye in 1947 but had otherwise no significant previous illness. One sister had died with multiple sclerosis.

Physical examination revealed evidence of leftsided optic atrophy and right-sided detached retina. There appeared to be mild left facial weakness. There was left-sided continuous facial myokymia involving mainly the lower half of the face. There was a spastic tetraparesis with generalized hypereflexia, absent abdominal reflexes and bilateral extensor plantar responses. Vibration sense was depressed below the iliac crests bilaterally. There was cerebellar ataxia in all four limbs.

He was admitted for investigation on 20 January 1969 when the physical signs were unchanged and the myokymia was still present.

Routine laboratory tests, including blood count and ESR, and chest and skull X-rays were normal. His CSF manometrics were normal, protein $50 \mathrm{mg} /$ $100 \mathrm{ml}$, gammaglobulin $18 \%$ of total protein, Lange 2233210000 and it contained 9 lymphocytes/ $\mathrm{mm}^{3}$.

Electromyography revealed continuous, rapidly recurring, spontaneous action potentials, amplitude $50-150 \mu \mathrm{V}$, at a rate of $80 / \mathrm{sec}$, in the facial muscles on the left side, with a tendency towards rhythmicity.

The patient was not placed on any specific treatment and was followed up after discharge until April 1969, since when he has defaulted from followup. The facial myokymia disappeared after 3 months.

\section{Case 3 (H.R.I., 162677)}

This 44-year-old lady, who works in a grocer's shop, was first seen as an out-patient at the Combined Neurological Service, Hull Royal Infirmary, on 12 July 1971 with a history of dragging and heaviness of the left leg since June 1966, followed by unsteadiness of gait. The initial episode improved after 3 months, but she had been left with some continuing weakness of both legs and ataxia, which had progressed very slowly.

Physical examination revealed that she had undulating contractions of the muscles of the right side of the face, typical of myokymia. This affected mainly the orbicularis oris and buccinator muscles around the mouth. There was no evidence of facial paresis. She had increased tone and brisk deep reflexes in the lower limbs with bilaterally extensor plantar responses. She had a broad-based ataxic gait, and minimal ataxia in the arms.
She was admitted for investigation on 26 July 1971 when her physical signs were found to be unchanged and the facial myokymia had persisted.

Routine laborary tests, including blood count and ESR, and chest and skull X-rays were normal. Routine EEG studies were normal. The CSF manometrics were normal, protein $59 \mathrm{mg} / 100 \mathrm{ml}$, gammaglobulin $27 \%$ total protein, Lange 2233210000 , and it contained 1 red cell and 1 lymphocyte $/ \mathrm{mm}^{3}$.

Electromyography. A simultaneous EMG recording of the activity in the orbicularis oris muscle in the right and left sides of the upper lip respectively was carried out using concentric needle electrodes (Fig. 2). This clearly shows the contrast between the two sides, there being on the affected right side single high voltage spontaneous action potentials of amplitude around $100 \mu \mathrm{V}$ occurring rhythmically, at regular intervals against a background of low voltage muscle activity. As a further illustration of this, and in order to demonstrate that the spontaneous muscle activity was not of cortical origin, the activity from the facial electrodes was also recorded on an EEG machine simultaneously with an EEG recording from conventional surface scalp electrodes. Figure 3 shows the trace obtained. There appear to be several motor units firing repetitively, each having its own rhythmicity. The motor unit of largest amplitude $(70 \mu \mathrm{V})$ fires at 12 cycles/sec.

The day after the above recordings the facial myokymia disappeared, the total duration from the date it was first observed having been 17 days. The patient was discharged with a recommendation for a course of corticotrophin therapy and physiotherapy.

\section{Discussion}

Fasciculation and myokymia are two separate conditions, which have been confused in the past, and can be distinguished by their clinical and electromyographic features. Fasciculations appear clinically as brief twitches seen scattered in an irregular manner over the surface of the muscle and occurring at irregular intervals. Myokymia is a slower contraction seen over small bands or strips of muscles, occurring in a regular manner which gives an undulating or rippling appearance to the overlying skin. Electromyographically, fasciculation is characterized by spontaneous high voltage simple single action potentials firing at random, whereas myokymia is characterized by spontaneous bursts of single action potentials occurring rhythmically at lower amplitudes. Each burst may consist of one to several individual action potentials, the constant feature being that they occur rhythmically. Sometimes the bursts of action potentials may have a tetanic character (Gutman et al., 1969).

Only single cases or very small series of patients with facial myokymia have so far been reported in 


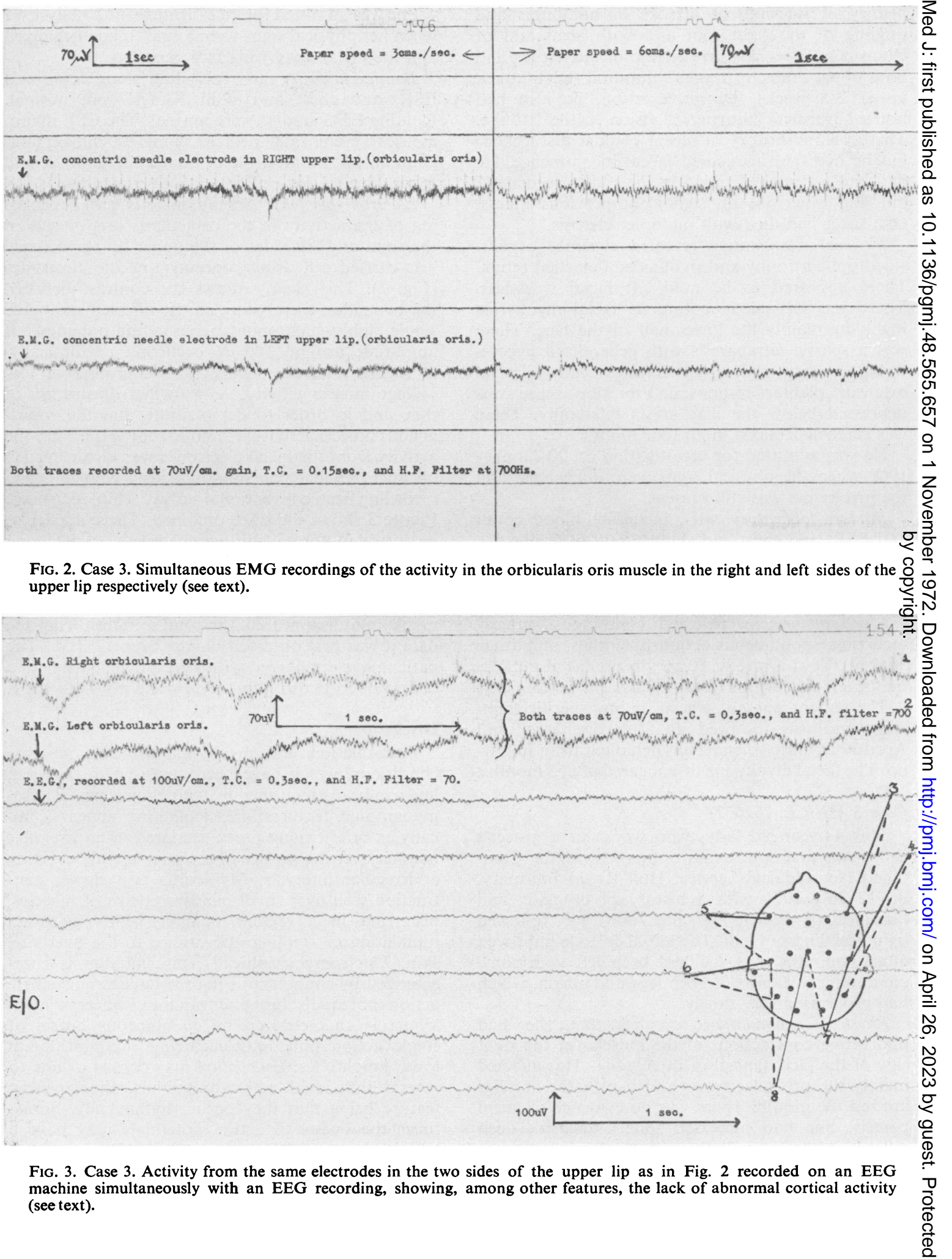


the literature. Among the most noteworthy of these is the report of Anderman et al. (1961) who described four personal cases and found seven in the literature. Matthews (1966) described six patients and found twelve in the literature, and the condition was reviewed in the British Medical Journal (1966). Gutman et al. (1969) described two patients and found forty in the literature. From these reports facial myokymia emerges as a rare phenomenon with distict characteristics.

Most patients affected by this condition were aware of the flickering sensation in one half of the face. In this respect all three of our patients are unusual in that throughout the course of their illness they were quite unaware of the abnormal movements. As in the patient described by Matthews, the onset was abrupt in Case 1 , and occurred quite suddenly whilst under observation, so that we can state that the movements were absent one day, and present the next. In addition to the flickering movements in the face, this patient showed clear evidence of a state of sustained muscle contraction on the affected side so that the palpebral fissure was narrowed and the lips were pursed. The result was a facial asymmetry, but there was no definite facial weakness. The undulating movement affected all the muscles innervated by the facial nerve, in Cases 1 and 2 , but they were most evident at the inner angle of the eye, the cheek and the angle of the mouth. It is of interest that the twitching movements were present continuously day and night, and persisted during sleep.

A significant feature of facial myokymia associated with multiple sclerosis is that it is almost invariably transient. In the hitherto reported cases it has persisted from 2 days to 3 months, with the exception of one case, in whom it did not disappear until the end of 6 months. In the present cases it lasted 17-30 days, but since the onset was uncertain in two patients the total duration is not known in them. Occasional instances of recurrence have been described and this can be either on the same side or on the opposite side of the face. Another significant fact is that appreciable facial weakness has not been a feature in those cases associated with multiple sclerosis. In only one of our patients (Case 2) was there any apparent facial weakness and this too was mild. The above features form a contrast to those seen in the reported cases of facial myokymia associated with pontine tumours (Espinosa, Lambert \& Klass, 1967) where significant and progressive facial paralysis was often present and where, in addition, progressing signs of other cranial nerve involvement and of pyramidal and cerebellar lesions were often present. Furthermore, in these cases the phenomenon tended to be persistent and has been reported to have been present for up to 13 years.
The clinical and electrical findings clearly indicate a lesion in the pons, and in the present cases there can be no doubt that the lesion was a plaque of demyelination adjacent to the facial nucleus. It is probable that the pathological process lies not in the nucleus but in the intra-axial part of the nerve, or in its supranuclear pathway. The involvement of the trigeminal fibres in two patients of Anderman et al. (1961) and in two of Matthews' (1966) cases may place the responsible lesion at a more rostral level in the pons.

Despite the later occurrence of focal epilepsy in one of our patients, there is no evidence that the myokymia is of cerebral cortical origin. The speed of the myokymia is much faster, and the amplitude much smaller than that observed in epilepsia partialis continua, and there has been no suggestion of any type of epilepsy in the other cases of facial myokymia described in the literature.

The relationship of facial myokymia to multiple sclerosis is important. It is clear that in the twentythree patients in the literature and in the present three cases that a causal relationship obtains. The phenomenon in the present patients developed during exacerbations of multiple sclerosis. In the vast majority of patients in whom facial myokymia has been transient and in whom there has been no significant facial paresis, the phenomenon has been associated with multiple sclerosis. The nature of the pathological process in the few without clinical evidence of multiple sclerosis remains obscure. It is of course possible that these patients too may suffer from multiple sclerosis, the myokymia being the first evidence of demyelination. Most of these patients are in the age-group most vulnerable to multiple sclerosis, but further episodes of neurological disease have not appeared in periods varying from 5 to 6 years in three of Matthews' patients. Only a more prolonged period of observation, and subsequent pathologic studies can clarify this issue.

\section{Acknowledgments}

We are grateful to Miss Patricia Willey and Miss Judith Crook for the electrophysiological tracings which are reproduced.

\section{References}

Anderman, F., Cosgrove, J.B.R., Lloyd-Smith, D.L., Gloor, P. \& McNaughton, F.L. (1961) Facial myokymia in multiple sclerosis. Brain, 84, 31.

British Medical Journal (1966) Leading Article. 2, 189.

ESPINOSA, R.E., LAMBERT, E.H. \& Klass, D.W. (1967) Facial myokymia affecting the electroencephalogram. Mayo Clinic Proceedings, 42, 258.

Gutman, L., Thompson, H.G. \& Martin, J.D. (1969) Transient facial myokymia. Journal of the American Medical Association, 209, 389.

Matthews, W.B. (1962) Epilepsy and disseminated sclerosis. Quarterly Journal of Medicine, 31, 141.

MATTHEWS, W.B. (1966) Facial myokymia. Journal of Neurology, Neurosurgery and Psychiatry, 29, 35. 


\section{Addendum}

Since this report was accepted for publication, we have encountered a fourth case:

Case 4 (H.R.I., 191412)

This 21-year-old female telephonist was admitted to the Combined Neurological Service, Hull Royal Infirmary, on 4 August 1972 with a 2-week history of numbness and a sensation of 'pins and needles' of the right side of the face and of the trunk and limbs on the left side. This was accompanied by vertigo, nausea, vomiting, deafness of the right ear, diplopia, unsteadiness of gait and a little weakness of the left arm and leg. These symptoms had been of rather rapid onset. The nausea, vomiting and vertigo had improved, but the other symptoms had progressed over this period. On direct questioning she said that over the past week she had noticed, while looking in the mirror, that there was a flickering of the skin immediately below the right eye.

About 2 months previously she had experienced the same sensory disturbance in the right of her face, deafness of the right ear and diplopia. These symptoms had been milder than in the present attack and had cleared up spontaneously in about 10 days. There was no other relevant past history and no relevant family history.

Physical examination revealed right-sided trigeminal sensory loss in all three divisions with a very sluggish corneal reflex on that side. There was a complete right-sided sixth cranial nerve palsy.
There was mild lateral nystagmus, worse on looking to the right. There were continuous, rhythmic, undulating involuntary movements, typical of facial myokymia, on the right side, involving the lower half of the orbicularis oculi, buccinator and orbicularis oris. There was no facial paresis. There was moderate right-sided nerve deafness. There was mild weakness of pyramidal distribution in the left arm and leg with an equivocal left plantar response, and impairment of all modalities of superficial sensation on the left side of the trunk, arm and leg. Her gait was mildly ataxic.

Routine laboratory tests, including blood count and ESR, chest and skull X-rays, and an EEG were normal. Her CSF manometrics were normal, protein $84 \mathrm{mg} / 100 \mathrm{ml}$, with a gammaglobulin of $21 \%$ and 2 lymphocytes $/ \mathrm{mm}^{3}$; Lange 1233221000 .

Electromyography revealed continuous, rapidly recurring, spontaneous action potentials, of amplitude $75-200 \mu \mathrm{V}$, in the facial muscles of the right malar region with a tendency towards rhythmicity. There were several motor units firing repetitively, in bursts, each having its own rhythmicity. The motor unit of largest amplitude $(200 \mu \mathrm{V})$ fired at 10 cycles/sec. At a corresponding point on the left side there were no spontaneous action potentials seen.

The patient was started on corticotrophin and bs the fourth day of therapy, apart from the facia $\vec{b}$. myokymia, only a mild right-sided nerve deafnes remained. 